\title{
Relación de la dieta con los niveles plasmáticos de lípidos y lipoproteínas en sujetos adultos con diferentes genotipos del gen de la apolipoproteína $\mathbf{E}$
}

\author{
Luisa Fernanda Tobar-Vargas ${ }^{1 *}$, Ana Lucía Torres y Martha Guerra \\ ${ }^{l}$ Pontificia Universidad Javeriana. Profesora Facultad de Ciencias. Depto. de Nutrición y Bioquímica. \\ Carrera 7 \# 43-82 Edificio Félix Restrepo (50) piso 3, Bogotá, Colombia. \\ ${ }^{2}$ Biología Molecular y Celular, Québec - Canadá. \\ *1tobar@javeriana.edu.co
}

Recibido: 08-04-2008; Aceptado: 26-06-2009

\begin{abstract}
Resumen
Objetivo. Establecer la posible relación entre dieta habitual, perfil lipídico y los genotipos de ApoE. Materiales y métodos. Participaron 150 profesores de la Pontificia Universidad Javeriana, se evaluó perfil lipídico, estado nutricional y porcentaje de grasa corporal. Otras variables: edad, género, polimorfismo del gen ApoE, estado de salud y consumo de alimentos. Resultados. 76 normolipidémicos (NL) y 74 hiperlipidémicos (HL). Los valores promedio de colesterol total (CT), LDLc y triglicéridos (TG) (mg/dl) fueron de 164,68 \pm 22,57; $91,82 \pm 23,39$ y $89,45 \pm 31,13$ para el grupo NL y de $223,67 \pm 20,25 ; 145,25 \pm 19,99$ y $198,74 \pm 49,95$ para el grupo HL. Edad promedio

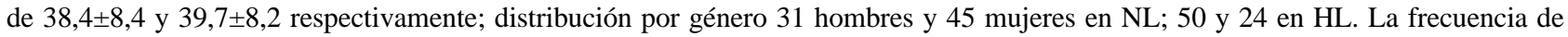
genotipos de la ApoE, fue similar a la reportada en otras poblaciones a nivel mundial. La evaluación del consumo de energía y nutrientes mostró una ingesta mayor que la recomendación para todos los nutrientes en la población en general, excepto para el consumo de colesterol en mujeres hiperlipidémicas. Conclusión. Al relacionar el consumo, el perfil lipídico y los genotipos, no se encontraron diferencias significativas en los niveles de lípidos y lipoproteínas pero sí tendencias en el grupo con genotipo 4/3 a tener niveles más altos de CT en comparación con los otros genotipos, especialmente en el grupo de HL.
\end{abstract}

Palabras clave: apolipoproteína E, consumo de alimentos, estado nutricional, genotipo, perfil lipídico, polimorfismo.

\begin{abstract}
Relationship between diet and serum levels of lipids and lipoproteins in adults with different apolipoprotein E genotypes. Objective. To find a possible relationship between: usual diet, lipid profile and ApoE genotypes. Materials and methods. Assessment of lipid profile, nutritional status and body fat percentage was conducted on 150 lecturers of the Pontificia Universidad Javerian a. Other variables were: age, gender, polymorphism of the ApoE gen, health status and food consumption. Results. 76 subjects were normolipidemic (NL) and 74 were hyperlipidemic (HL). Total cholesterol (TC), LDLc and triglycerid (TG) average values (mg/dl) were 164.68 \pm 22.57 ; $91.82 \pm 23.39$ and $89.45 \pm 31.13$ respectively for the NL group, and $223.67 \pm 20.25 ; 145.25 \pm 19.99$ and $198.74 \pm 49.95$ respectively for the HL group. Average age was $38.4 \pm 8.4$ for NL and 39.7 \pm 8.2 for HL; gender distribution was of 31 men and 45 women in NL, and 50 men and 24 women in the HL group. ApoE genotype frequency was similar to that reported in other populations around the world. The evaluation of energy and nutrient consumption revealed an intake higher than the overall recommendation for all the nutrients, except cholesterol in HL women. Conclusion. When correlating consumption, lipid profile and genotypes, no significant differences were found, neither in lipid nor lipoprotein levels. However, a tendency was observed in the $4 / 3$ genotype to be prone to higher cholesterol levels compared to other genotypes, especially in the HL group.
\end{abstract}

Key words: apolipoprotein E, food consumption, nutritional status, genotype, lipid profile, polymorphism. 


\section{Resumo}

Relação da dieta com os níveis plasmáticos de lipídeos e lipoproteínas em indivíduos adultos com diferentes genótipos do gene da apolipoproteína E. Objetivo. Estabelecer a possível relação entre dieta habitual, perfil lipídeo e os genótipos ApoE. Materiais e métodos. Participaram 150 professores da "Pontificia Universidad Javeriana", avaliou-se o perfil lipídeo, o estado nutricional e o percentual de gordura corporal. Outras variáveis: idade, sexo, polimorfismo do gene ApoE, saúde e consumo de alimentos. Resultados. 76 normolipidémicos (NL) e 74 hiperlipidémicos (HL). Os valores médios de colesterol total (CT), LDLc e triglicérides (TG) (mg / dl) foram 164,68 \pm 22,57; 91,82 $\pm 23,39$ e 89,45 $\pm 31,13$ para o grupo NL e 223,67 $\pm 20,25 ; 145,25 \pm 19,99$ e 198,74 \pm 49,95 para o grupo HL. Idade média de 38,4 \pm 8,4 e 39,7 $\pm 8,2$, respectivamente; distribuição por sexos 31 homens e 45 mulheres na NL; 50 e 24 em HL. A frequiência dos genótipos da ApoE foi semelhante ao reportado em outras populações em todo o mundo. A avaliação do consumo de energia e nutrientes apresentou maior assimilação do que a recomendação para todos os nutrientes na população em geral, exceto para o consumo de colesterol em mulheres hiperlipidémicas. Conclusão. Relacionando o consumo, o perfil lipídeo e os genótipos, não foram observadas diferenças significativas nos níveis de lipídeos e de lipoproteínas, mas se as tendências no grupo com genótipo 4/3 para ter níveis mais elevados de CT em comparação com outros genótipos, especialmente no grupo HL.

Palavras chave: apolipoproteína E, consumo de alimentos, estado nutricional, genótipo, perfil lipídico, polimorfismo.

\section{Introducción}

La enfermedad cardiovascular (ECV) es una de las causas principales de morbilidad, discapacidad y muerte prematura que a nivel mundial alcanza cifras alarmantes. En Colombia es la segunda causa de enfermedad y muerte para hombres y mujeres mayores de 45 años según lo reportó el Ministerio de la Protección Social en 2003 (1). La ECV es una entidad multicausal, con muchos factores de riesgo establecidos. Entre éstos existen unos no modificables como la edad, género y la historia familiar de enfermedad cardiovascular prematura y otros modificables entre los que se cuentan el estilo de vida de cada persona como, por ejemplo, el hábito alimentario, el hábito de fumar, presencia de obesidad, hipertensión, estrés, inactividad física, diabetes mellitus y parámetros bioquímicos como los niveles de colesterol total, colesterol-LDL (LDL-C) y de HDL$\mathrm{C}(2)$.

Los niveles séricos de colesterol están influenciados por el consumo de alimentos y especialmente la composición de la grasa dietaria. Frente a las variaciones en el consumo de alimentos, se ha visto que no todos los individuos tienen la misma sensibilidad para desarrollar alteraciones lipídicas. De ahí que se deba tener en cuenta otros aspectos, tales como los genéticos. Las variaciones en los niveles plasmáticos de colesterol están en estrecha relación con diversas enzimas, receptores y apolipoproteínas que participan en el metabolismo de las lipoproteínas. El metabolismo depende de factores genéticos que pueden hacer variar los valores.

Existen diferentes isoformas de las apoliproteínas entre las cuales la ApoE presenta un gen polimórfico, con tres alelos codominantes $\varepsilon 2, \varepsilon 3$ y $\varepsilon 4$ que dan origen a tres genotipos homocigotos E2/2, E3/3 y E4/4 y a tres heterocigotos E3/2, E4/3 y E4/2 (3). Estos alelos se diferencian por la sustitución de un aminoácido en las posiciones 112 y 158. El genotipo E2 presenta una cisteína en ambos alelos, el E3 tiene una cisteína en la posición 112 y una arginina en la 158, mientras que el E4 tiene argininas en las dos posiciones (4).

La presencia de estos alelos puede tener efectos sobre los niveles de lípidos séricos y por tanto se considera como uno de los factores de riesgo genético para el desarrollo de la aterosclerosis. En personas sanas, más del $10 \%$ de la variación interindividual normal en los niveles de colesterol, puede ser debida a este polimorfismo de la ApoE (5). Los hallazgos sugieren que los individuos con el alelo $\varepsilon 4$ tienen niveles más altos de colesterol total y de colesterol LDL y así también presentan mayor riesgo cardiovascular, mientras que el alelo $\varepsilon 2$ presenta niveles más bajos. En Colombia se llevó a cabo un estudio para la determinación del polimorfismo de la ApoE en la población bogotana normolipidémica y los hallazgos fueron parecidos; los individuos con el alelo $\varepsilon 4$ presentaron niveles de colesterol que a pesar de estar en el rango normal $(<200 \mathrm{mg} / \mathrm{dl})$, tenían los valores más cercanos a este rango, en comparación con los otros genotipos (6).

Teniendo en cuenta que la alimentación desempeña un papel fundamental, modulando el balance homeostático de múltiples procesos fisiológicos que se asocian directamente con interacción genética, es importante profundizar en el conocimiento de estas relaciones; por esta razón se estudió la relación del consumo de alimentos habitual, con la respuesta del perfil lipídico y la presencia del polimorfismo de la ApoE. 


\section{Materiales y métodos}

Se trabajó con una población de 150 profesores de planta de la Pontificia Universidad Javeriana. Adultos entre $20 \mathrm{y}$ 65 años, sin tratamientos médicos que afectarán los niveles de lípidos plasmáticos, o en tratamiento dietario, sin diabetes mellitus, hipotiroidismo, hipertiroidismo o enfermedades que afectaran los niveles de lípidos sanguíneos. Las variables dependientes que se evaluaron fueron del perfil lipídico: concentración del colesterol total (CT), colesterol LDL (LDL-C), colesterol HDL (HDL-C), triglicéridos (TG), colesterol VLDL (VLDL-C); el estado nutricional se evaluó por del índice de masa corporal (IMC), peso corporal en $\mathrm{kg}$, estatura en metros y el porcentaje de grasa corporal, evaluado por la toma de 6 pliegues corporales (tríceps, subescapular, supraespinal, abdominal, muslo y pierna), e interpretados mediante la utilización de la fórmula de Yuhasz (7). Se tomaron en cuenta las variables de edad en años, género, polimorfismo del gen ApoE, estado de salud y el consumo de alimentos, como independientes.

Para la toma de muestras se tuvieron en cuenta las condiciones preanalíticas recomendadas mundialmente para este tipo de determinaciones como: ayuno previo de 12 horas, instrucciones de evitar ejercicios o estrés durante las 24 horas anteriores a la toma de muestra y no presentar modificaciones recientes en su peso.

Se realizó una toma de sangre mediante venopunción directa utilizando tubos vacutainer secos. La sangre se centrifugó a $3000 \mathrm{rpm}$ por 15 minutos para separar el suero y el plasma. A partir del suero se determinó colesterol, sus fracciones y triglicéridos por métodos enzimáticos (Laboratorios Bayer S.A.). El colesterol VLDL se calculó mediante la fórmula de Friedewald teniendo en cuenta que las muestras no tuvieron niveles de triglicéridos mayores a $400 \mathrm{mg} / \mathrm{dL}(8)$.

El DNA genómico se extrajo a partir de los leucocitos de sangre periférica mediante el kit Wizard Genomic DNA Purification (Promega A1120).

Para la genotipificación por $P C R-R F L P$ (Polymerase Chain Reaction - Restriction Fragment Legth Polymorphism) de las isoformas comunes de la apolipoproteína E (E2, E3, E4), se utilizaron dos oligonucleótidos: F6: 5' TAA-GCTTGG-CAC-GGC-TGT-CCA-AGG-A 3’ y F4: 5’ ACA-GAATTC-GCC-CCG-GCC-TGG-TAC-AC 3' descritos por Hixon y Vernier (1990), para amplificar el fragmento de 244 pb en el exón 4 del gen de la apolipoproteína E. La amplificación se realizó en un termociclador (BIO-RAD MyCycler).
Para determinar las variaciones específicas que causan el polimorfismo en el gen de la ApoE, los productos de amplificación se sometieron a digestión con la enzima de restricción HhaI, seguido por separación electroforética en gel de poliacrilamida $10 \%(\mathrm{p} / \mathrm{v})$ no desnaturalizante, y visualización de los fragmentos sobre una fuente de luz ultravioleta.

\section{Recolección de la información sobre salud, nutrición y alimentación}

Todos los sujetos firmaron un consentimiento informado aceptando participar en el estudio. Se aplicó la Encuesta de Salud, Nutrición y Alimentación para los Empleados de la Pontificia Universidad Javeriana, retomada del programa de estilos de vida saludable (9).

Se indagó sobre aspectos como información general, antecedentes médicos personal y familiares y estilo de vida y frecuencia de consumo de alimentos, de la cual se obtuvo información sobre el consumo aproximado de energía y nutrientes de cada persona. El consumo se comparó con la recomendación de energía y nutrientes para hombres y mujeres adultos colombianos propuestas por el Instituto Colombiano de Bienestar Familiar (10). Se calculó el porcentaje de adecuación y se reportó como individuos en riesgo cuando su consumo estuvo por debajo del $66,6 \%$ de la recomendación, para efectos de este artículo se menciona como "<RDA", (por su sigla en inglés, Recommended Dietary Adequacy) la cual se interpretó como menor de $66,6 \%$ de RDA, entre $66,6 \%$ y $100 \%$ como "RDA" y cuando es mayor al $100 \%$ se menciona como "> RDA" (11).

Se realizó la toma de datos antropométricos para determinar la valoración nutricional de todos los individuos. Los instrumentos que se utilizaron para este fin fueron: para la toma del peso, una balanza de pie marca Detecto con capacidad de $150 \mathrm{~kg}$, para la estatura un estadiómetro marca Height $200 \mathrm{~cm}$ No. 26 SM, para la toma de perímetros una cinta métrica marca Seca y para los pliegues cutáneos, se utilizó un adipómetro marca Holtain - Tanner. Para la toma de los pliegues y circunferencias se utilizaron las normas de Lohman (12) y para la interpretación del porcentaje de grasa corporal los datos ajustados de Lohman, 1987 (13).

\section{Manejo estadístico de la información}

El manejo estadístico de los datos, se realizó en Excel y con el programa Statistix 7. La determinación de la interrelación entre los factores analizados se efectuó mediante un análisis de varianza (ANOVA), que permitió com- 
parar los niveles de lípidos y lipoproteínas entre los grupos genotípicos definidos por el polimorfismo $\varepsilon 2 \varepsilon 3 \varepsilon 4 \mathrm{del}$ gen de la ApoE. En los grupos donde se encontraron diferencias significativas se aplicó la prueba para comparación de medias de Tukey, esta prueba arrojó un valor crítico Q indicativo de que cuando hay valores por encima de éste se rechaza la hipótesis nula de que las medias son iguales, o sea que hay diferencia significativa entre los grupos ( $p$ $<0,05)$.

Cuando se necesitaba comparar las medias entre dos grupos tales como los niveles de colesterol total entre hombres y mujeres, se utilizó la prueba $t$ para dos muestras suponiendo varianzas desiguales, ya que en estos casos esta prueba tiene una mejor potencia y se evitan los errores tipo 1 .

\section{Resultados}

La muestra tuvo una distribución por género así: 81 hombres y 79 mujeres. La edad promedio en los hombres fue de 39,4 y en las mujeres de 38,8 años.

\section{Valores del perfil lipídico}

Los niveles promedio para toda la población fueron: CT de 182,92 mg/dl, TG de 128,2 mg/dl, LDL-C de 106,57 mg/dl, VLDL-C de 25,63 mg/dl y HDL-C de 47,34 mg/dl. De los 150 individuos estudiados, 76 eran normolipidémicos, y 74 dislipidémicos. De estos últimos, 26 presentaron hipercolesterolemia aislada, 27 hipertrigliceridemia y 21 dislipidemia mixta.

En la tabla 1 se puede observar la clasificación del perfil lipídico según el promedio de los niveles de lípidos y lipoproteínas sanguíneas que presentó la población. En general en los normolipidémicos, se observan valores si- milares en las fracciones lipídicas tanto en hombres como en mujeres, se destaca que los TG y el VLDL-C en las mujeres son menores que en el grupo de hombres, mientras que el HDL-C es más alto en las mujeres. Al observar la población con dislipidemia mixta, se encontró un valor de triglicéridos y VLDL-C mayor en hombres que en mujeres.

\section{Antecedentes de salud personales y familiares}

Las características generales en cuanto a los antecedentes de salud tanto personales como familiares, hábitos de vida saludables y hábitos alimentarios presentaron un comportamiento similar entre los grupos de normolipidémicos e hiperlipidémicos. Sin embargo, es importante resaltar que los hiperlipidémicos presentan, en general, porcentajes un poco mayores en todas las variables.

\section{Valoración nutricional}

Los promedios y la desviación estándar de los datos obtenidos de la toma de peso, estatura, perímetros de cintura y cadera se encuentran en la tabla 2.

La relación cintura/cadera se utiliza como factor predictor de riesgo a desarrollar enfermedad cardiovascular. Se encontraron valores normales tanto en los hombres como en las mujeres ( $<1$ y $<0,8$ respectivamente). Se identificó que la mayor parte de la población, el $56,7 \%$, tiene un IMC normal. Esta población está compuesta por 72,5\% mujeres y $43,2 \%$ hombres. En general un 38\% presentó sobrepeso, de este el $27,6 \%$ y el $49,4 \%$, corresponden a mujeres y hombres respectivamente. Sólo el 1,3\% presentó bajo peso y un $4 \%$ obesidad grado I.

La fórmula de Yuhaz se aplicó para identificar el porcentaje de grasa corporal, se encontró que la mayoría de los hombres presentaron un porcentaje de grasa corporal bue-

Tabla 1. Concentración promedio de lípidos y lipoproteínas de la población normolipidémica $(\mathrm{N}=76)$ y con dislipidemia mixta $(\mathrm{N}=74)$

\begin{tabular}{lcccccc}
\hline \multirow{2}{*}{ Fracción lipídica } & \multicolumn{3}{c}{ Normolipidémica $(\mathbf{N = 7 6 )}$} & \multicolumn{2}{c}{ Dislipidemia mixta (N=74) } \\
\cline { 2 - 7 } & Hombres & Mujeres & Ambos géneros & Hombres & Mujeres & Ambos géneros \\
\hline Colesterol total mg/dl & $165,08 \pm 21,69$ & $164,41 \pm 23,40$ & $164,68 \pm 22,57$ & $224,87 \pm 20,96$ & $212,25 \pm 2,47$ & $223,67 \pm 20,25$ \\
Triglicéridos mg/dl & $103,10 \pm 27,79$ & $80,06 \pm 30,07$ & $89,45 \pm 31,13$ & $202,76 \pm 50,82$ & $160,50 \pm 13,44$ & $198,74 \pm 49,95$ \\
LDL-C mg/dl & $93,71 \pm 22,38$ & $90,51 \pm 24,22$ & $91,82 \pm 23,39$ & $146,42 \pm 20,70$ & $134,15 \pm 2,33$ & $145,25 \pm 19,99$ \\
VLDL-C mg/dl & $20,62 \pm 5,56$ & $15,99 \pm 5,56$ & $17,88 \pm 6,23$ & $40,56 \pm 10,17$ & $32,10 \pm 2,69$ & $39,75 \pm 9,99$ \\
HDL-C mg/dl & $49,06 \pm 8,77$ & $55,89 \pm 6,97$ & $53,11 \pm 8,40$ & $34,71 \pm 5,30$ & $44,25 \pm 1,06$ & $35,62 \pm 5,79$ \\
\hline
\end{tabular}


Tabla 2. Promedio y desviación estándar de los datos antropométricos de la población estudiada ( $\mathrm{N}=150)$

\begin{tabular}{lccc}
\hline Medida antropométrica & Hombres & Mujeres & Ambos géneros \\
\hline Peso $(\mathbf{k g})$ & $74,74 \pm 9,89$ & $57,73 \pm 6,76$ & $66,91 \pm 12,07$ \\
Estatura $(\mathbf{m})$ & $1,72 \pm 0,06$ & $1,57 \pm 0,06$ & $1,65 \pm 0,09$ \\
Índice de masa corporal & $25,40 \pm 3,11$ & $23,30 \pm 2,56$ & $24,44 \pm 3,05$ \\
Cintura $(\mathbf{c m})$ & $90,24 \pm 9,27$ & $74,06 \pm 7,28$ & $82,79 \pm 11,85$ \\
Cadera $(\mathbf{c m})$ & $98,38 \pm 5,64$ & $95,84 \pm 5,41$ & $97,21 \pm 5,66$ \\
Relación cintura/cadera & $0,92 \pm 0,06$ & $0,77 \pm 0,05$ & $0,85 \pm 0,09$ \\
\hline
\end{tabular}

no y las mujeres en igual proporción entre buena y aceptable. En relación con la grasa corporal la mitad de la población está clasificada como bueno $(51,3 \%)$, casi un $30 \%$ como aceptable y pocos tienen una composición atlética (12\%); algunos fueron reportados como regulares $(6 \%)$ y obesos (2\%).

\section{Evaluación de consumo de alimentos}

El promedio de consumo de energía y nutrientes se observa en la tabla 3. Al distribuir las poblaciones según su concentración de lípidos y el consumo de nutrientes, se observó que los hombres del grupo de normolipidémicos tienen un consumo de energía y nutrientes mayores en comparación con las mujeres normolipidémicas y con los hiperlipidémicos. Cuando se evalúa de forma general el consumo, el grupo de hiperlipidémicos tiene un consumo mayor en comparación con los normolipidémicos.

En la evaluación del porcentaje de adecuación, se encontró un consumo elevado de energía y nutrientes, los datos se observan en la tabla 4 . Al realizar porcentaje de adecuación por género y clasificación según su perfil lipídico, se observó que en el grupo de hombres todos tienen un con- sumo de energía y nutrientes por encima de la recomendación, aunque esa adecuación presenta porcentajes un poco más altos entre los normolipidémicos en comparación con los hombres hiperlipidémicos. En el grupo de las mujeres, también se presentó un consumo de nutrientes mayor a la recomendación, excepto en el colesterol que entre las mujeres hiperlipidémicas se encuentra en el rango adecuado en comparación con las mujeres normolipidémicas; para los demás nutrientes hay porcentajes mayores en las mujeres hiperlipidémicas.

\section{Distribución de los genotipos del polimorfismo del gen ApoE dentro de la población}

Con respecto al genotipo del gen ApoE, el más común fue el $3 / 3$ con una frecuencia de $66,0 \%$, seguido por el $22,6 \%$ del $4 / 3$, una frecuencia menor fue la de los $3 / 2$ del $8,0 \%$ y la menor frecuencia fue para los genotipos 4/4, 4/2 y $2 / 2$ con un 3,32\% (Figura 1, Tabla 5). En cuanto a las frecuencias de los alelos de la ApoE en la población estudiada, se encontró que el alelo $\varepsilon 3$ es el más común $(0,81 \mathrm{n}=244)$, seguido del $\varepsilon 4$ y $\varepsilon 2$, en ese orden $(0,13 \mathrm{n}=40$ y $0,05 \mathrm{n}=16)$ respectivamente, la frecuencia de alelos es contabilizada sobre $n=300$.

Tabla 3. Promedio de consumo de nutrientes en la población de normolipidémicos y de hiperlipidémicos

\begin{tabular}{lcccccc}
\hline Nutriente & \multicolumn{3}{c}{ Normolipidémicos } & & \multicolumn{2}{c}{ Hiperlipidémicos } \\
\cline { 2 - 7 } & Hombres & Mujeres & General & Hombres & Mujeres & General \\
\hline Energía (Kcal) & $2838,1 \pm 748,7$ & $2040,8 \pm 552,4$ & $2366 \pm 747,5$ & $2700,6 \pm 919,1$ & $2105,8 \pm 618,3$ & $2507,7 \pm 875,3$ \\
Proteína (g) & $113,9 \pm 38,4$ & $81,3 \pm 24,3$ & $94,6 \pm 34,6$ & $105,6 \pm 41,1$ & $81,7 \pm 26,9$ & $97,9 \pm 38,6$ \\
Grasa (g) & $114,5 \pm 39$ & $85,1 \pm 23,5$ & $97,1 \pm 33,9$ & $107,8 \pm 42,6$ & $87,8 \pm 26,7$ & $101,3 \pm 39,2$ \\
Colesterol (mg) & $406,3 \pm 216,2$ & $309 \pm 162,4$ & $348,7 \pm 191$ & $381 \pm 217,4$ & $289,7 \pm 162,4$ & $351,4 \pm 204,6$ \\
Carbohidratos $(\mathbf{g})$ & $347,4 \pm 98$ & $251,3 \pm 84,7$ & $290,5 \pm 101,5$ & $340,7 \pm 126$ & $257,4 \pm 85,2$ & $313,7 \pm 120,3$ \\
\hline
\end{tabular}


Tabla 4. Porcentaje de adecuación de energía y nutrientes en la población masculina y femenina normolipidémica e hiperlipidémica del estudio

\begin{tabular}{|c|c|c|c|c|c|}
\hline \multirow{3}{*}{ Nutriente } & \multicolumn{5}{|c|}{ Población masculina } \\
\hline & \multicolumn{2}{|c|}{ Consumo } & & \multicolumn{2}{|c|}{ \% Adecuación } \\
\hline & $\begin{array}{l}\text { Normoli- } \\
\text { pidémicos }\end{array}$ & $\begin{array}{l}\text { Hiperli- } \\
\text { pidémicos }\end{array}$ & Recomendado & $\begin{array}{l}\text { Normoli- } \\
\text { pidémicos }\end{array}$ & $\begin{array}{l}\text { Hiperli- } \\
\text { pidémicos }\end{array}$ \\
\hline Energía (Kcal) & $2838,1 \pm 748,7$ & $2700,6 \pm 919,1$ & 2194 & 129 & 123 \\
\hline Proteína (g) & $113,9 \pm 38,4$ & $105,6 \pm 41,1$ & 75 & 152 & 141 \\
\hline Grasa total (g) & $114,5 \pm 39$ & $107,8 \pm 42,6$ & 73 & 157 & 148 \\
\hline Colesterol (mg) & $406,3 \pm 216,2$ & $381 \pm 217,4$ & 300 & 135 & 127 \\
\hline \multirow[t]{2}{*}{ Carbohidratos (g) } & $347,4 \pm 98$ & $340,7 \pm 126$ & 309 & 112 & 110 \\
\hline & \multicolumn{5}{|c|}{ Población femenina } \\
\hline \multirow[t]{2}{*}{ Nutriente } & \multicolumn{2}{|c|}{ Consumo } & & \multicolumn{2}{|c|}{ \% Adecuación } \\
\hline & $\begin{array}{l}\text { Normoli- } \\
\text { pidémicos }\end{array}$ & $\begin{array}{l}\text { Hiperli- } \\
\text { pidémicos }\end{array}$ & Recomendado & $\begin{array}{l}\text { Normoli- } \\
\text { pidémicos }\end{array}$ & $\begin{array}{l}\text { Hiperli- } \\
\text { pidémicos }\end{array}$ \\
\hline Energía (Kcal) & $2040,8 \pm 552,4$ & $2105,8 \pm 618,3$ & 1710 & 119 & 123 \\
\hline Proteína (g) & $81,3 \pm 24,3$ & $81,7 \pm 26,9$ & 58 & 140 & 141 \\
\hline Grasa total (g) & $85,1 \pm 23,5$ & $87,8 \pm 26,7$ & 57 & 149 & 154 \\
\hline Colesterol (mg) & $309 \pm 162,4$ & $289,7 \pm 162,4$ & 300 & 103 & 97 \\
\hline Carbohidratos (g) & $251,3 \pm 84,7$ & $257,4 \pm 85,2$ & 241 & 104 & 107 \\
\hline
\end{tabular}

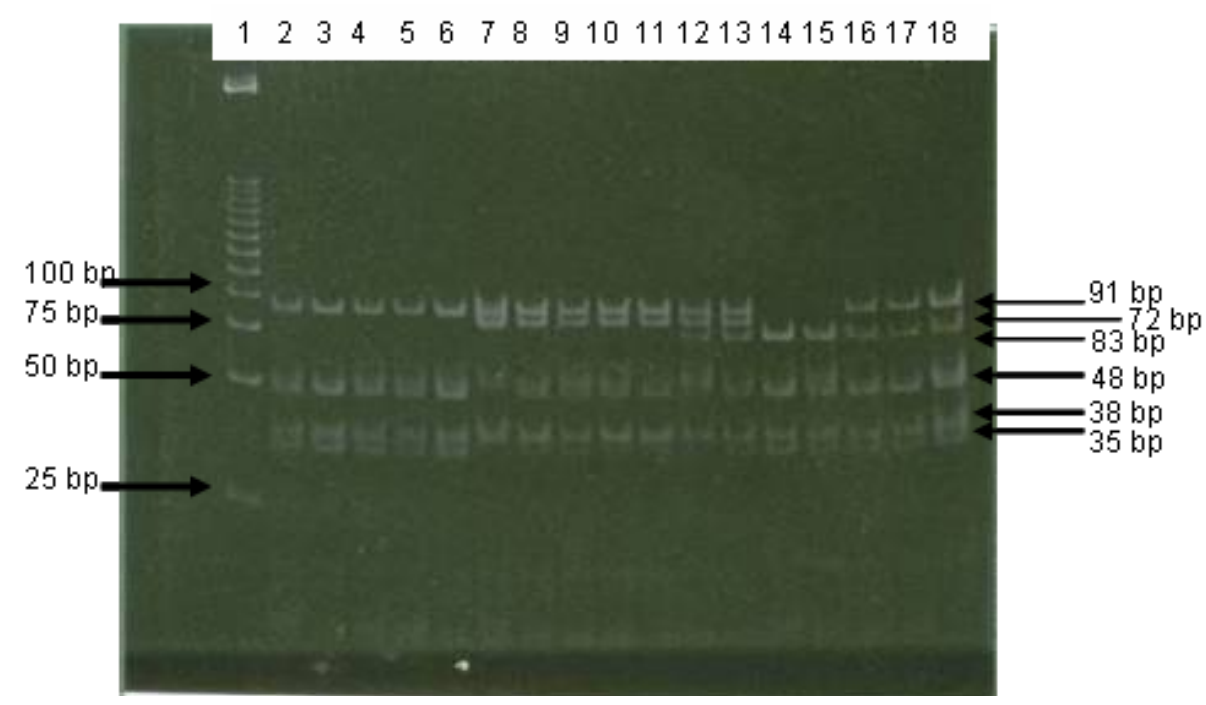

Figura 1. Gel de poliacrilamida al 10\% (p/v) con coloración de bromuro de etidio para identificar los genotipos del polimorfismo del gen de la ApoE. Carril 1: marcador de talla molecular 25 bp ladder; Carriles 2-6 genotipo Apo E3/3; Carril 7 genotipo apo E2/2; Carriles 8-11 genotipo Apo E3/2; Carriles 12-13 genotipo Apo E4/2; Carriles 14-15 genotipo Apo E4/4; Carriles 16-18 genotipo Apo E4/3. 
Tabla 5. Distribución de los genotipos del polimorfismo de la ApoE de la población según género

\begin{tabular}{lcccccc}
\hline Genotipo & \multicolumn{2}{c}{ Hombres } & \multicolumn{2}{c}{ Mujeres } & \multicolumn{2}{c}{ Ambos } \\
\hline & No. & \% & No. & $\%$ & No. & $\%$ \\
\hline $2 / 2$ & 1 & 0,66 & 0 & 0 & 1 & 0,66 \\
$3 / 2$ & 6 & 4 & 6 & 4 & 12 & 8 \\
$3 / 3$ & 53 & 35,33 & 46 & 30,66 & 99 & 66 \\
$4 / 2$ & 2 & 1,33 & 0 & 0 & 2 & 1,33 \\
$4 / 3$ & 18 & 12 & 16 & 10,66 & 34 & 22,66 \\
$4 / 4$ & 1 & 0,66 & 1 & 0,66 & 2 & 1,33 \\
Total & 81 & 54 & 69 & 46 & 150 & 100 \\
\hline
\end{tabular}

\section{Asociación de los resultados con los diferentes genotipos encontrados del gen ApoE}

Los análisis de los niveles de lípidos y lipoproteínas de acuerdo a los genotipos del gen de la ApoE se presentan en la tabla 6. Los valores promedio de los lípidos y lipoproteínas para los sujetos normolipidémicos presentaron una tendencia a niveles de CT, TG, LDL-C y VLDL-C más altos para el grupo de sujetos con el genotipo $3 / 3$ en comparación con el resto de grupos, aunque esta tendencia no representa una diferencia significativa (Tabla 6).

Se realizaron los análisis separados por género y por genotipo de ApoE, en estas comparaciones de perfiles lipídicos se observó que en el grupo E3/3 los hombres tienen niveles más altos de TG, VLDL-C y más bajos de HDL-C comparado con las mujeres E3/3, los valores fueron significativos cuando se hizo la prueba de comparación entre medias de Tukey, donde el valor crítico Q es de 4,55 , para los TG este valor fue de 5,58 lo que indica que se rechaza la hipótesis de que las medias son iguales ya que hubo una diferencia significativa $(p<0,05)$.

Para los valores promedio de los lípidos y lipoproteínas para los sujetos hiperlipidémicos, se observó en particular que los sujetos con genotipo $2 / 2,4 / 2$ y $4 / 4$ que tuvieron niveles de TG más elevados, los datos no son comparables por el bajo número de sujetos con estos genotipos. Los valores de CT fueron mayores para el grupo con genotipo $4 / 3$ en comparación con los otros aunque esta tendencia no representa una diferencia significativa (Tabla 6).

Además se realizaron los análisis separados por género y por genotipo de ApoE, en estas comparaciones de perfiles lipídicos se vio que en el grupo E3/3 los hombres tienen niveles más altos de TG, VLDL-C y más bajos de HDL-C comparado con las mujeres E3/3, para el genotipo 4/3, los hombres también presentaron niveles de HDL-C mucho menores que las mujeres, los valores fueron significativos. Cuando se hizo la prueba de comparación entre media de Tukey, el valor crítico Q fue de 4,74, para las HDL-C entre hombres y mujeres E3/3 este valor fue de 6,52 y para los 4/ 3 fue de 5,48 lo que indica una diferencia significativa ( $p$ $<0,05)$.

\section{Asociación de los genotipos del gen de la ApoE con el consumo de energía total y grasa total}

Se evaluó el consumo de alimentos, en este caso el consumo de energía total y grasa total para observar el comportamiento del perfil lipídico según el genotipo.

En el grupo de personas normolipidémicas no se hicieron comparaciones para los niveles de lípidos y lipoproteínas según los genotipos y la adecuación de energía menor de la RDA porque el número de personas, para algunos genotipos, fue muy pequeño. Al hacer la comparación de los niveles de CT entre los genotipos E3/2, E3/3 y E4/3 en los sujetos con un porcentaje de adecuación de energía igual a la RDA no hubo diferencias significativas entre los genotipos; de forma similar, tampoco hubo diferencias significativas en los sujetos con adecuación mayor que RDA (Tabla 7).

Al comparar los niveles de TG entre los genotipos, no se encontraron diferencias significativas para los sujetos con una adecuación de energía igual o mayor a la RDA. Sin 
Tabla 6. Concentración de lípidos y lipoproteínas según los genotipos del gen de la ApoE en los individuos normolipidémicos e hiperlipidémicos

\begin{tabular}{|c|c|c|c|c|c|c|c|c|c|c|c|c|}
\hline \multirow{2}{*}{$\begin{array}{l}\text { Fracción } \\
\text { lipídica } \\
\text { (mg/dl) }\end{array}$} & \multicolumn{5}{|c|}{ Normolipidémicos } & \multirow[b]{2}{*}{$4 / 4$} & \multirow[b]{2}{*}{$2 / 2$} & \multicolumn{5}{|c|}{ Hiperlipidémicos } \\
\hline & $2 / 2$ & $3 / 2$ & $3 / 3$ & $4 / 2$ & $4 / 3$ & & & $3 / 2$ & $3 / 3$ & $4 / 2$ & $4 / 3$ & $4 / 4$ \\
\hline Col - total & 0 & $145,1 \pm 31,6$ & $167,5 \pm 22,7$ & 0 & $162,1 \pm 16$ & 145 & 153 & $205,2 \pm 30$ & $200,3 \pm 28,6$ & $144,3 \pm 15,9$ & $211,6 \pm 42,1$ & 213,5 \\
\hline TG & 0 & $66,7 \pm 9,1$ & $93,6 \pm 33,5$ & 0 & $84 \pm 21,4$ & 55,5 & 182,5 & $131,2 \pm 49$ & $174,6 \pm 70,6$ & $192,8 \pm 49,9$ & $162,1 \pm 63,4$ & 181,5 \\
\hline LDL-C & 0 & $70,1 \pm 34,7$ & $94,4 \pm 22,5$ & 0 & $90,8 \pm 20,3$ & 72 & 81,2 & $127 \pm 32,6$ & $121,2 \pm 31,1$ & $71,5 \pm 21,9$ & $127,4 \pm 45,5$ & 140,5 \\
\hline VLDL-C & 0 & $13,3 \pm 1,9$ & $18,7 \pm 6,7$ & 0 & $16,7 \pm 4,3$ & 11,1 & 36,5 & $26,2 \pm 9,8$ & $34,9 \pm 14,1$ & $38,6 \pm 10$ & $32,4 \pm 12,7$ & 36,3 \\
\hline HDL-C & 0 & $60,2 \pm 7,4$ & $52,4 \pm 8,3$ & 0 & $52,8 \pm 8,7$ & 60 & 33,3 & $49,6 \pm 11,5$ & $40 \pm 9,9$ & $32 \pm 2,8$ & $43,6 \pm 13,1$ & 33,5 \\
\hline Total & 0 & 5 & 55 & 0 & 15 & 1 & 1 & 7 & 44 & 2 & 19 & 1 \\
\hline
\end{tabular}

embargo, es de anotar que los niveles más altos de TG los presentaron los sujetos con el genotipo $3 / 3$ sin importar el nivel de consumo de energía (Tabla 7).

En relación con los niveles de LDL-C, en general, los sujetos con el genotipo 3/3 tuvieron los niveles más altos al compararlos con los 3/2 y 4/3. Para aquellos con una adecuación de energía igual a la RDA, no hubo diferencias significativas ( $\mathrm{p}=0,41)$, pero los valores más altos los presentaron los sujetos con genotipo 3/3. Para los sujetos con una adecuación del consumo de energía mayor que la RDA, tampoco hubo diferencias significativas entre grupos $(\mathrm{p}=0,40)$. Los genotipos $3 / 3$ y $4 / 3$ presentaron niveles de LDL-C similares y para el 3/2 fueron más bajos (Tabla 7).

Para la fracción VLDL-C no se encontró diferencia significativa entre los sujetos con adecuación de energía igual a la RDA, quienes tuvieron datos muy similares entre los genotipos $3 / 3$ y 4/3. Para los sujetos con adecuación de energía mayor que la RDA tampoco hubo diferencias significativas, sin embargo, los valores más bajos para VLDLC los presentó el grupo de genotipo 3/2 (Tabla 7).

Tabla 7. Distribución de los niveles promedios de lípidos y lipoproteínas según el genotipo del gen de la ApoE y la adecuación del consumo de energía total en personas normolipidémicas.

\begin{tabular}{|c|c|c|c|c|c|c|c|c|c|}
\hline \multirow{3}{*}{$\begin{array}{l}\text { Fracción lipídica } \\
\text { (mg/dl) }\end{array}$} & \multicolumn{8}{|c|}{ Genotipos } & \\
\hline & \multicolumn{3}{|c|}{$3 / 2$} & \multicolumn{3}{|c|}{$3 / 3$} & \multicolumn{3}{|c|}{$4 / 3$ y $4 / 4$} \\
\hline & $<\mathrm{RDA}$ & RDA & $>$ RDA & $<$ RDA & RDA & $>$ RDA & $<$ RDA & RDA & $>$ RDA \\
\hline Col-Total & 135 & & $147,6 \pm 35,9$ & 173,5 & $171,7 \pm 18,7$ & $166,2 \pm 24,0$ & & $158,2 \pm 15,8$ & $162,3 \pm 16,7$ \\
\hline $\mathrm{TG}$ & 76 & & $64,4 \pm 8,6$ & 129,0 & $83,8 \pm 30,8$ & $95,6 \pm 34,1$ & & $76,1 \pm 22,0$ & $85,0 \pm 22,3$ \\
\hline LDL-C & 57,5 & & $73,3 \pm 39,2$ & 80,5 & $100,0 \pm 17,4$ & $93,2 \pm 23,9$ & & $81,6 \pm 15,0$ & $93,3 \pm 21,8$ \\
\hline VLDL-C & 15,2 & & $12,8 \pm 1,8$ & 25,8 & $16,8 \pm 6,2$ & $19,1 \pm 6,8$ & & $15,2 \pm 4,4$ & $16,9 \pm 4,4$ \\
\hline HDL & 60,0 & & $60,3 \pm 8,5$ & 66,0 & $52,9 \pm 8,0$ & $51,9 \pm 8,2$ & & $60,1 \pm 8,1$ & $50,2 \pm 7,2$ \\
\hline Total personas & 1 & & 4 & 1 & 12 & 42 & $\mathbf{0}$ & 5 & 11 \\
\hline
\end{tabular}

$*<\mathrm{RDA}=<66,6 \%, \mathrm{RDA}=66,6 \mathrm{y}<100 \% \mathrm{y}>\mathrm{RDA}=>100 \%$ 
Cuando se compararon los niveles de HDL-C entre los genotipos, no se encontraron diferencias significativas en los sujetos con porcentaje de adecuación de energía igual o mayor que la RDA. Los sujetos 4/3 con energía igual que la RDA presentaron valores mayores que los del grupo 3/3 (Tabla 7).

En el grupo de personas normolipidémicas no se presentaron personas con porcentaje de adecuación de consumo de grasa total más bajo que la RDA. En los sujetos con un porcentaje de adecuación de grasa total igual a la RDA no se hicieron comparaciones para los niveles de lípidos y lipoproteínas según los genotipos, porque el número de personas es muy pequeño. En los sujetos con un consumo de grasa total mayor que la RDA no hubo diferencias significativas $(\mathrm{p}=0,70)$ al comparar los niveles de CT entre los genotipos. Se evidenció que los valores de CT en los grupos 3/3 y 4/3 fueron más altos que los del grupo 3/2. Resultados similares se observaron al analizar los niveles de LDL-C (Tabla 8).

Las concentraciones más altas de TG y VLDL-C los presentaron los sujetos con el genotipo 3/3 y una adecuación de grasa mayor que la RDA. Por otro lado, los individuos con genotipo $4 / 3$ presentaron los niveles más bajos. En cuanto al HDL-C, en los sujetos con genotipo 3/2 se observaron niveles más altos aunque las diferencias no fueron significativas (Tabla 8).

También se analizaron los niveles de lípidos según la adecuación de energía y grasa total por genotipo del gen de la ApoE, en el grupo de personas hiperlipidémicas.
Para los niveles de lípidos y lipoproteínas, no se hicieron comparaciones según los genotipos y la adecuación de energía menor de la RDA porque el número de personas fue pequeño.

Al hacer la comparación de los niveles de CT y LDL-C entre los genotipos E3/2, E3/3 y E4/3 en los sujetos con un porcentaje de adecuación de energía igual que la RDA, no se obtuvieron diferencias significativas, pero se observó una tendencia a presentar niveles de CT y LDL-C más altos en los sujetos con el genotipo 4/3. Un resultado interesante en los sujetos con adecuación de energía mayor que la RDA e hiperlipidémicos, fue la diferencia significativa en el CT entre los genotipos 4/3 y 4/2 (en la prueba de Tukey de comparación entre medias, el valor crítico de $\mathrm{Q}$ fue de 4,25 y para el CT este valor fue de 4,48 lo que indica que se rechaza la hipótesis nula que dice que las medias son iguales), se comprobó con el $t$-test, prueba de mayor robustez cuando se comparan sólo dos medias, el nivel de significancia de $\mathrm{p}=0,30$ entre estos 2 grupos, esto sugiere que no hay diferencia entre estos grupos (Tabla 9).

Al comparar los niveles de TG entre los genotipos, no se encontraron diferencias significativas para los sujetos con una adecuación de energía igual que la RDA; los niveles más altos se observaron en el genotipo 4/3, los cuales fueron similares a los observados en los genotipos $3 / 2$ y $3 / 3$. En los sujetos con adecuación de energía mayor que la RDA, tampoco hubo diferencias significativas, pero los niveles más altos de TG los presentaron los sujetos con el genotipo 4/2 y los más bajos fueron los del genotipo $3 / 2$ y $2 / 2$, datos no comparables por ser grupos muy pequeños (Tabla 9).

Tabla 8. Distribución de los niveles promedio de lípidos y lipoproteínas según el genotipo del gen de la ApoE y la adecuación del consumo de grasa total en personas normolipidémicas.

\begin{tabular}{|c|c|c|c|c|c|c|c|c|c|}
\hline \multirow{3}{*}{$\begin{array}{l}\text { Fracción lipídica } \\
\text { (mg/dl) }\end{array}$} & \multicolumn{9}{|c|}{ Genotipos } \\
\hline & \multicolumn{3}{|c|}{$3 / 2$} & \multicolumn{3}{|c|}{$3 / 3$} & \multicolumn{3}{|c|}{$4 / 3$ y $4 / 4$} \\
\hline & $<$ RDA & RDA & $>$ RDA & $<$ RDA & RDA & $>$ RDA & $<$ RDA & RDA & $>$ RDA \\
\hline Col-Total & & 135 & $147,6 \pm 35,9$ & & 173,5 & $167,4 \pm 22,9$ & & $164,8 \pm 27,9$ & $160,5 \pm 15,3$ \\
\hline TG & & 76 & $64,4 \pm 8,6$ & & 129 & $93,0 \pm 33,5$ & & $80,8 \pm 35,7$ & $82,4 \pm 21,3$ \\
\hline LDL-C & & 57,5 & $73,3 \pm 39,2$ & & 80,5 & $94,7 \pm 22,6$ & & $86 \pm 19,8$ & $90,2 \pm 21$ \\
\hline VLDL-C & & 15,2 & $12,8 \pm 1,8$ & & 25,8 & $18,6 \pm 6,7$ & & $16,2 \pm 7,1$ & $16,4 \pm 4,2$ \\
\hline HDL & & 60,0 & $60,3 \pm 8,5$ & & 66 & $52,2 \pm 8,1$ & & $59 \pm 1,4$ & $52,5 \pm 8,9$ \\
\hline Total personas & $\mathbf{0}$ & 1 & 4 & $\mathbf{0}$ & 1 & 54 & $\mathbf{0}$ & 2 & 14 \\
\hline
\end{tabular}


Para la fracción VLDL-C no se encontró diferencia significativa entre los sujetos con adecuación de energía igual que la RDA. Para los sujetos con adecuación de energía mayor que la RDA tampoco hubo diferencias significativas, sin embargo, los valores más bajos para VLDL-C los presentó el grupo de genotipo 3/2 y 2/2 (Tabla 9).

Al comparar los niveles de HDL-C entre los genotipos, no se encontraron diferencias significativas entre los sujetos con porcentaje de adecuación de energía igual al RDA y tampoco para los sujetos con adecuación de energía mayor que la RDA; sin embargo los niveles más altos de HDL$\mathrm{C}$ se presentaron en el grupo con genotipo $3 / 2$ y consumo mayor que la RDA (Tabla 9).

Los análisis de los niveles de lípidos de acuerdo al genotipo y la adecuación de grasa para el CT y el LDL-C en los sujetos con un porcentaje de adecuación de grasa igual al RDA no mostraron diferencias significativas entre los genotipos, se observó una similitud en los valores entre los genotipos $3 / 3$ y 4/3. Un dato interesante de resaltar es que los sujetos con adecuación de grasa mayor que la RDA e hiperlipidémicos, presentaron una diferencia significativa en el CT entre los genotipos 4/3 y 4/2 (en la prueba de Tukey de comparación entre medias, el valor crítico de $Q$ fue de 4,16 y para el CT este valor fue de 4,70 lo que indica que se rechaza la hipótesis nula que dice que las medias son iguales), se comprobó con el ttest y un nivel de significancia $\mathrm{p}=0,28$ entre estos 2 grupos, lo cual sugiere que en definitiva no hay diferencia entre estos grupos (Tabla 10).

En la comparación de los niveles de TG y VLDL-C entre los genotipos $3 / 2,3 / 3$ y $4 / 3$, en los sujetos con un porcentaje de adecuación de grasa igual al RDA, los valores fue- ron mayores para los sujetos con genotipo 4/3, mientras que cuando el consumo de grasa fue mayor que la RDA, los valores más altos los presentaron los sujetos con genotipo $3 / 3$ y los más bajos los $3 / 2$ (Tabla 10).

En los niveles de HDL-C no se observaron diferencias significativas entre genotipos en los diferentes niveles de adecuación de grasa. En general los valores más altos correspondieron al grupo con genotipo 3/2 (Tabla 10).

\section{Discusión}

Al analizar el perfil lipídico de la población teniendo en cuenta su género, se observó que las concentraciones medias de CT, LDL-C, TG y VLDL-C fueron mayores en el grupo de hombres que en el de mujeres, mientras que las de HDL-C fueron mayores en las mujeres, esto muestra que en esta población (edad promedio de 39,4 \pm 8,3 hombres y $38,8 \pm 8,5$ mujeres) las mujeres tienen un mejor perfil lipídico que los hombres, especialmente antes de llegar a la menopausia. Tendencia similar describe la Sociedad Española de Arteriosclerosis (1994), (14) que encontró que las mujeres adultas tienen niveles mayores de HDL-C mientras que los hombres presentan mayores niveles de CT y LDL-C, lo cual lleva a tener mayor riesgo de desarrollar ECV. Es interesante ver como al separar la población en normolipidémica e hiperlipidémica, se ve la misma tendencia en los hombres a presentar un perfil lipídico más alto que el de las mujeres.

Los diferentes estudios acerca de la frecuencia de alelos del gen de la ApoE a nivel mundial, muestran la variabilidad entre las etnias. La tendencia general a nivel mundial es que el genotipo E3/3 es el más común entre 50 y $70 \%$

Tabla 9. Distribución de los niveles promedios de lípidos y lipoproteínas según el genotipo del gen de la ApoE y la adecuación del consumo de energía total en personas hiperlipidémicas.

\begin{tabular}{|c|c|c|c|c|c|c|c|c|c|c|c|}
\hline $\begin{array}{l}\text { Fracción } \\
\text { lipídica } \\
\text { (mg/dl) }\end{array}$ & $3 / 2$ y $2 / 2$ & & & $3 / 3$ & Genotipos & & $4 / 3$ y $4 / 4$ & & & $4 / 2$ & \\
\hline$<$ RDA & RDA & $>$ RDA & $<$ RDA & RDA & $>$ RDA & $<$ RDA & RDA & $>$ RDA & $<$ RDA & RDA & $>\mathrm{RDA}$ \\
\hline Col-Total & $187,8 \pm 42,0$ & $205,2 \pm 30,4$ & $200,3 \pm 19,4$ & $200,1 \pm 38,9$ & $200,3 \pm 27,4$ & 201,5 & $214,9 \pm 47,7$ & $210,2 \pm 39,8$ & & & $144,3 \pm 15,9$ \\
\hline TG & $155,0 \pm 69,3$ & $127,2 \pm 37,5$ & $176,8 \pm 9,5$ & $151,0 \pm 52,8$ & $179,2 \pm 75,3$ & 136,5 & $162,8 \pm 61,0$ & $165,6 \pm 67,8$ & & & $192,8 \pm 49,9$ \\
\hline LDL-C & $110,0 \pm 48,3$ & $128,0 \pm 27,1$ & $122,9 \pm 18,2$ & $121,5 \pm 35,5$ & $121,1 \pm 31,4$ & 125,2 & $131,9 \pm 48,2$ & $125,5 \pm 45,9$ & & & $71,5 \pm 21,9$ \\
\hline VLDL-C & $31,0 \pm 13,9$ & $25,4 \pm 7,5$ & $35,4 \pm 1,9$ & $30,2 \pm 10,6$ & $35,8 \pm 15,1$ & 27,3 & $32,6 \pm 12,2$ & $33,1 \pm 13,6$ & & & $38,6 \pm 10,0$ \\
\hline HDL & $44,7 \pm 10,0$ & $49,3 \pm 14,0$ & $37,5 \pm 8,5$ & $44,9 \pm 15,0$ & $39,1 \pm 8,79$ & 47,0 & $46,9 \pm 14,8$ & $41,8 \pm 12,3$ & & & $32,0 \pm 2,8$ \\
\hline Total personas 0 & 3 & 5 & 2 & 7 & 35 & 1 & 8 & 11 & 0 & 0 & 2 \\
\hline
\end{tabular}

$*<\mathrm{RDA}=<66,6 \%, \mathrm{RDA}=66,6 \mathrm{y}<100 \% \mathrm{y}>\mathrm{RDA}=>10$ 
Tabla 10. Distribución de los niveles promedio de lípidos y lipoproteínas según el genotipo del gen de la ApoE y la adecuación del consumo de grasa total en personas hiperlipidémicas

\begin{tabular}{|c|c|c|c|c|c|c|c|c|c|c|c|c|}
\hline \multirow{3}{*}{$\begin{array}{l}\text { Fracción lipídica } \\
(\mathrm{mg} / \mathrm{dl})\end{array}$} & \multicolumn{12}{|c|}{ Genotipos } \\
\hline & \multicolumn{3}{|c|}{$3 / 2$ y $2 / 2$} & \multicolumn{3}{|c|}{$3 / 3$} & \multicolumn{3}{|c|}{$4 / 3$ y $4 / 4$} & \multicolumn{3}{|c|}{$4 / 2$} \\
\hline & $<$ RDA & RDA & $>$ RDA & $<$ RDA & RDA & $>$ RDA & $<$ RDA & RDA & $>$ RDA & $<$ RDA & RDA & $>$ RDA \\
\hline Col-Total & & 169 & $202,9 \pm 33,6$ & & $203,9 \pm 33,5$ & $199,4 \pm 27,8$ & 201,5 & $210,8 \pm 46,2$ & $212,7 \pm 42,3^{*}$ & & & $144,3 \pm 15,9$ \\
\hline TG & & 218,5 & $126,1 \pm 39,2$ & & $165,4 \pm 53,0$ & $176,7 \pm 74,5$ & 136,5 & $183,9 \pm 62,6$ & $157,5 \pm 64,4$ & & & $192,8 \pm 49,9$ \\
\hline LDL-C & & 90,5 & $125,7 \pm 34,5$ & & $124,4 \pm 29,6$ & $120,5 \pm 31,8$ & 125,2 & $128,7 \pm 51,6$ & $128,0 \pm 45,4$ & & & $71,5 \pm 21,9$ \\
\hline VLDL-C & & 43,7 & $25,2 \pm 7,8$ & & $33,1 \pm 10,6$ & $35,3 \pm 14,9$ & 27,3 & $36,8 \pm 12,5$ & $31,5 \pm 12,9$ & & & $38,6 \pm 10,0$ \\
\hline HDL & & 33,2 & $49,6 \pm 11,5$ & & $42,9 \pm 15,1$ & $39,3 \pm 8,5$ & 47,0 & $42,9 \pm 17,2$ & $42,9 \pm 12,3$ & & & $32,0 \pm 2,8$ \\
\hline Total personas & 0 & 1 & 7 & 0 & 8 & 36 & 1 & 5 & 14 & 0 & 0 & 2 \\
\hline
\end{tabular}

$*<\mathrm{RDA}=<66,6 \%, \mathrm{RDA}=66,6 \mathrm{y}<100 \% \mathrm{y}>\mathrm{RDA}=>100 \%$

aproximadamente, la población estudiada presentó una frecuencia del $66 \%$. En su orden los genotipos $4 / 3$ y $3 / 2$ son comunes en la población estudio $22,6 \%$ y $8 \%$, mientras que el E4/4 es poco común y los E2/2 y el heterocigoto E4/2 son muy escasos, tal y como se ve reflejado en lo hallado $1,33 \%, 0,66 \%$ y $1,33 \%$ respectivamente; lo anterior es comparable con poblaciones como Islandia, Finlandia, Hungría, China, India (15).

Al comparar la distribución de alelos para la población estudiada se encontró que la frecuencia del $\varepsilon 3(0,81)$ es similar a la reportada en la población de Japón y Turquía $(0,84 ; 0,88)(15)$, Marruecos y Korea $(0,85 ; 0,87)(16-18)$, ligeramente más alta que la población de Alemania $(0,77)$, Estados Unidos $(0,76)$, y un poco más baja que la de Finlandia $(0,69)$ y Sudán $(0,61)(15)$. La frecuencia del alelo $\varepsilon 4$ fue de 0,13 , similar a las poblaciones de Islandia, Hungría, India y Malasia $(0,16 ; 0,13 ; 0,13$ y 0,12$)$, mayor a la de China y Japón $(0,74)$ y menor que la de Finlandia y Sudán $(0,24 ; 0,29)(15)$. Los valores del $\varepsilon 2$ fueron de 0,05 en la población estudio, estos valores son similares a los de la población de India, Islandia, Finlandia y Hungría $(0,04 ; 0,06 ; 0,06 ; 0,06)$ y más bajos que los de Japón y Sudán $(0,08 ; 0,08)(15)$.

Numerosos estudios acerca del efecto del polimorfismo de la ApoE sobre el metabolismo de lípidos reportan que los individuos que tienen el alelo $\varepsilon 2$ presentan una tendencia a tener niveles plasmáticos de CT más bajos que individuos homocigotos con alelo $\varepsilon 3$, mientras que los sujetos con al menos un alelo $\varepsilon 4$ tienden a tener los niveles más altos de CT que los homocigotos $\varepsilon 3$ (5). Para la población estudio normolipidémica se encontraron datos de CT lige- ramente más elevados en el grupo $3 / 3$ que en el $4 / 3$, mientras que los 3/2 los tuvieron los niveles de CT más bajos; mientras que en el grupo de hiperlipidémicos para el genotipo 4/3 el comportamiento fue similar al reportado por Davignon, pero discrepan en cuanto los valores promedio de CT en el grupo con genotipo $3 / 2$, ya que fueron ligeramente mayores que para los $3 / 3$.

Otros estudios han analizado el papel de las interacciones entre genes y nutrientes en respuesta a los niveles de los lípidos plasmáticos así como las variaciones en el consumo de grasa, de colesterol o de ambos y los resultados han sido contradictorios (19-21). En el presente estudio, se observaron los niveles promedio de lípidos y lipoproteínas según el genotipo del gen de la ApoE y el porcentaje de adecuación de consumo de energía y grasa total habitual, para los sujetos normolipidémicos y los hiperlipidémicos, resultados que también presentaron algunas contradicciones.

En general no se encontraron diferencias significativas, pero sí se observaron niveles de CT más altos en los sujetos normolipidémicos con genotipo $3 / 3$ y $4 / 3$ con adecuación de energía mayor que la RDA y más bajos en los del genotipo 3/2. Mientras que según la adecuación de grasa total mayor que la RDA los niveles de CT fueron mayores para los $3 / 3$ que para los $4 / 3$, la misma situación se observó para los niveles de LDL-C; esta situación es contraria a la reportada en algunos estudios al considerar el efecto del genotipo sobre el perfil lipídico (5). En general los sujetos normolipidémicos con genotipo $3 / 3$ y consumo de energía mayor que de la RDA tuvieron valores más elevados para CT, TG, LDL-C y VLDL-C en comparación con los 4/ 
3 , mientras que los sujetos con genotipo $3 / 2$ presentaron los valores más bajos para las mismas fracciones y a su vez los más altos para HDL-C en comparación con los otros genotipos.

Para los sujetos hiperlipidémicos tampoco se presentaron diferencias significativas. Los valores de CT más elevados se observaron en los sujetos 4/3 con una adecuación de energía igual a la RDA, en comparación con los otros genotipos, e incluso con aquellos que tuvieron una adecuación de energía mayor que la RDA.

En los sujetos hiperlipidémicos, los valores de CT y LDLC, fueron más altos para el genotipo 4/3 y 4/4 en comparación con los otros, esto puede indicar que en esta población el genotipo 4/3 de la ApoE ejerce su efecto en el aumento de los niveles de colesterol de una manera más pronunciada cuando los sujetos tiene una adecuación de grasa mayor a la RDA, un comportamiento similar al descrito por Utermann, 1987, quien encontró que en comparación con otros genotipos, los individuos que portaban el alelo $\varepsilon 4$ tenían los niveles plasmáticos más altos de CT y LDL-C. Es de destacar que si a estos individuos se les suministra una dieta baja en grasa, hay una disminución más grande en sus niveles de CT y LDL que la que sucede en los individuos con genotipos $3 / 3$ y $3 / 2$. Esto ha sido demostrado en estudios como los realizados por Sarkkinen, et al. (1998), (22), quienes hicieron una modificación a los aportes de grasa total y colesterol y confirmaron que el genotipo de la ApoE tiene un efecto sobre la respuesta de los lípidos ante las variaciones de las dietas e incluso reportaron variaciones mayores para el alelo $\varepsilon 4$ cuando se modificaba sólo la grasa dietaria.

Los niveles de TG y VLDL-C en el grupo de normolipidémicos con porcentaje de adecuación de energía mayor e igual a la RDA y de grasa mayor a la RDA, presentaron valores mayores para los sujetos con el genotipo $3 / 3$ en comparación con los 4/3. En el grupo de hiperlipidémicos con porcentaje de adecuación mayor que la RDA para energía, los valores más altos fueron para el grupo con genotipo $4 / 2$ y $3 / 3$.

En los individuos hiperlipidémicos con porcentaje de adecuación de grasa mayor que la RDA se observó que los sujetos con el genotipo 4/2 tuvieron los valores más altos y en su orden siguieron los $3 / 3$ y $4 / 3$; los valores más bajos se presentaron en los sujetos con genotipo $3 / 2$, aquí se ve como la asociación del alelo $\varepsilon 2$ con un factor ambiental de riesgo (consumo de grasa elevado) y uno genético (presencia del alelo ع4) contribuye a un aumento de los TG. Estos datos fueron similares a los reportados por Erkkilä et al., en 2001 (23) quienes realizaron un estudio en el cual hicieron variaciones en la dieta en cuanto al aporte de grasa y carbohidratos (sacarosa), encontrando que al variar los carbohidratos, se presentaba una tendencia al aumento en los niveles de TG para los sujetos con alelo $\varepsilon 2$. Por el contrario, en un estudio realizado en adultos normolipidémicos realizado por Weintraub et al., 1987 (24) el alelo $\varepsilon 2$ se asoció con incremento en los niveles posprandiales de triglicéridos.

Al realizar comparaciones de grupos con el mismo nivel de clasificación del porcentaje de adecuación de nutrientes y el mismo genotipo se observó que presentaron diferencias significativas en los valores de lípidos, para citar un caso, en los individuos normolipidémicos e hiperlipidémicos con genotipo 3/3 cuya adecuación de energía fue mayor que la RDA, hubo diferencias significativas en los valores de CT entre estos dos grupos. Lo mismo se observó al hacer los análisis con la adecuación del consumo de grasa; estas variaciones pueden sugerir que en esos sujetos hiperlipidémicos lo que causa el aumento de las fracciones lipídicas está relacionado con otras causas de tipo genético o ambiental (aparte del genotipo de la ApoE y del consumo de grasa y energía) que deberían ser estudiadas con mayor profundidad. Este comportamiento también se observó para las fracciones de TG, VLDL-C y HDL-C en quienes tuvieron consumo igual a la RDA y mayor que la RDA para energía y se hicieron repetitivas al tener en cuenta la adecuación de grasa total, según el genotipo para los valores promedio de las fracciones de CT, TG, HDL-C, LDL-C y VLDL-C.

De acuerdo con esto, los estudios que han analizado la relación entre el gen de la ApoE y los nutrientes (genmedio ambiente) muestran que esta interacción se ve más claramente cuando los sujetos son hiperlipidémicos, en tanto que es más difícil detectar diferencias entre los distintos genotipos ApoE en los sujetos o poblaciones normolipidémicas (25). Los problemas relacionados con las variaciones y contradicciones en los resultados de los diferentes estudios pueden ser debidos a otros factores asociados como son el tamaño de la muestra, efectos de la edad, del género, estado de menopausia, índice de masa corporal, niveles iniciales de lípidos y lipoproteínas de los participantes al iniciar los estudios, diferencias étnicas y culturales en relación con la dieta, las variaciones de los protocolos de estudio y la dificultad para asegurar la recopilación de toda la información $(26,27)$, especialmente la de evaluación de consumo de energía y nutrientes. 


\section{Conclusión}

Cuando se analiza el perfil lipídico y el genotipo de la ApoE, se observa una tendencia en los sujetos E4/E3 a presentar niveles más altos de CT, especialmente entre los hiperlipidémicos. Los resultados de este trabajo en su conjunto, a pesar de que no aportan una evidencia significativa de la relación entre el consumo de alimentos y la respuesta del perfil lipídico de acuerdo a los genotipos de la ApoE, sugieren, que existe una tendencia a tener niveles más altos de colesterol-LDL cuando el consumo de energía y grasa total es igual a la recomendación, en el grupo con genotipo E4/E3. Adicionalmente, se confirma que el efecto de los genotipos de la ApoE se nota más claramente en las situaciones patológicas que en las personas con condiciones normales de perfil lipídico.

\section{Agradecimientos}

A los profesores de planta de tiempo completo y medio tiempo de las diferentes facultades, por su participación como grupo de estudio. Finalmente, a las estudiantes Ana María Gelvis Consuegra y Francy Milena Jiménez Boada, estudiantes de trabajo de grado de la carrera de Bacteriología por la toma y el procesamiento de las muestras sanguíneas para determinar los perfiles lipídicos. Al Dr. Leonardo Lareo por la revisión de este escrito.

\section{Financiación}

Proyecto financiado por la Pontificia Universidad Javeriana, número de registro del proyecto fue el 1589.

\section{Conflicto de intereses}

Los autores declaran que no existen conflictos de intereses con los resultados de este trabajo.

\section{Referencias}

1. Ministerio de Protección Social, Instituto Nacional de Salud. Boletín epidemiológico semanal. SIVIGILA. Sistema Nacional de Salud Pública en Colombia. Indicadores básicos de salud en Colombia. 2003. http:/ / w w w .col.ops-oms . org/sivigila/2003/ BOLE38_03.htm Consultado: junio 2005

2. Peyser PA. Genetic epidemiology of coronary artery disease. Epidemiology Review 1997; 19: 80-90.

3. Mahley RW, Huang Y and Rall SC Jr. Pathogenesis of type III Hyperlipoproteinemia (dysbetalipoproteine- mia): questions, quandaries and paradoxes. Journal Lipid Research 1999; 40: 1933-1949.

4. Rall SC, Weisgraber KH, Mahley RW. Human apolipoprotein E: the complete amino acid sequence. Journal Biology Chemistry 1982; 257: 4171-4178.

5. Davignon J, Gregg RE, Sing CF. Apolipoprotein E polymorphisms and atherosclerosis. Artherosclerosis 1988; 8: 1-21.

6. Torres AL. Fundación para la promoción de la investigación y la tecnología, Banco de la República. Niveles de colesterol total y colesterol-LDL en individuos de Santa Fe de Bogotá. Influencia del polimorfismo de la apolipoproteína E. Editorial La Fundación. Bogotá, Colombia. 2001; 87 p.

7. Yuhasz MS. Physical fitness Manual. University of Western Ontario. London, Ontario.

8. Friedewald WT, Levy RI, and Fredrickson DS. Estimation of the concentration of low density lipoprotein cholesterol in plasma, without use of the separative ultracentrifuge. Clinical Chemistry 1972; 18: 499-502.

9. Díaz M. Programa de estilos de vida saludable. Pontificia Universidad Javeriana, Bogotá, 2002.

10. I.C.B.F. Recomendaciones de consumo diario de calorías y nutrientes para la población Colombiana. I.C.B.F. Bogotá, Colombia. 1990; 73 p.

11. Gibson RS. Principles of nutritional assessment. Oxford University Press. New York, USA. 1990; 147148.

12. Lohman TG, Roche AF, Martorell R. Anthropometric standardization reference manual. Abridged edition. Illinois, USA. 1991; 3-8, 44-46, 53, 55-70.

13. Esparza F. Manual de cineantropometría. FEMEDE. España. 1993; 162.

14. Sociedad Española de Arteriosclerosis, Sociedad Española de Medicina Interna y Liga de la Lucha contra la Hipertensión Arterial. Recomendaciones para la prevención primaria de la enfermedad cardiovascular. Science Investigation Artherosclerosis. 1994; 6: 62-102.

15. Hallman M, Boerwinkle E, Saha N, Sandholzer Ch, Menzel HJ, Csázár A and Utermann G. The apolipoprotein E polymorphism: A comparison of allele frequencies and effects in nine populations. American Journal Human Genetics 1991; 49: 338-339. 
16. Zekraoui L, Lagarde JP, Raisonnier A, et al. High frequency of the apolipoprotein E 4 allele in African pygmies and most of the African populations in SubSaharan Africa. Human Biology 1997; 69: 575-581.

17. Valveny N, Esteban E, Kandil M, et al. APOE polymorphism in Spanish and Moroccan populations. Clinical Genetics 1997; 51: 354-356.

18. Wu YN, Zhang JW, Zhang ZX, et al. An association analysis of apolipoprotein E genotypes with Alzheimer's disease in Chinese population. Zhongguo Yi Xue Ke Xue Yuan Xue Bao 2001; 23: 450-454.

19. Tall A, Welch C, Applebaum-Bowden D, Wassef M and the Working Group. Interaction of diet and genes in atherogenesis. Report of an NHLBI Working Group Artheriosclerosis. Thrombosis Vascular Biology 1997; 17: 3326-3331.

20. Ordovas JM, Schaefer EJ. Genes, variation of cholesterol and fat intake and serum lipids. Current Opinion Lipidology 1999; 10: 15-22.

21. Ordovas JM. The genetics of serum lipid responsiveness to dietary interventions. Proceedings of the Nutrition Society 1999; 58: 171-187.

22. Sarkkinen E, et al. Effect of apolipoprotein E polymorphism on serum lipid response to the separate modification of dietary fat and dietary cholesterol. American Journal of Clinical Nutrition 1998; 68: 1215-1222.

23. Erkkilä A, et al. Apo E polymorphism and the hypertryglyceridemic effect of dietary sucrose. American Journal of Clinical Nutrition 2001; 73: 746-752.

24. Weintraub MS, Eisenberg S, Breslow JL. Dietary fat clearance in normal subjects is regulated by genetic variation in apolipoprotein E. Journal Clinical Investigation 1987; 80: 1571-1577.

25. Berglund L. 2004. APO E: ¿Un candidato importante para la interacción entre genes y nutrientes? Department of Medicine, University of California Davis, Junio Consultado: http://www.siicsalud.com/ dato/dat042/05222000.htm. Consultado en marzo 2005 .

26. Ye Sh and Kwiterovich J. Influence of genetic polymorphisms on responsiveness to dietary fat and cholesterol. American Journal of Clinical Nutrition 72(suppl): 2000; 1275S-1284S.

27. Mansson LF, McNeill G and Avenell A. Genetic variation and the lipid response to dietary intervention: a systematic review. American Journal Clinical Nutrition 2003; 77: 1098-1111. 